\title{
Identification and Sensitivity of Vaginal and Probiotic Lactobacillus species to Urinary Antibiotics
}

\author{
Lipika Singhal ${ }^{1}$ Varsha Gupta ${ }^{1}$ Menal Gupta ${ }^{1}$ Poonam Goel ${ }^{2} \quad$ Jagdish Chander ${ }^{1}$ \\ ${ }^{1}$ Department of Microbiology, Government Medical College and \\ Hospital, Chandigarh, India \\ 2Department of Obstetrics and Gynaecology, Government Medical \\ College and Hospital, Chandigarh, India

\begin{abstract}
Address for correspondence Menal Gupta, MBBS, MD, Department of Microbiology, Government Medical College and Hospital, Sector 32, Chandigarh 160030, India (e-mail: m.gupta1013@gmail.com).
\end{abstract}

J Lab Physicians:2020;12:111-114

\begin{abstract}
Objectives Healthy vaginal microbiota is mainly dominated by Lactobacillus species namely L. crispatus, L. gasseri, L. jensenii, and L. iners. Lactobacilli are thought to play an important role in the prevention of urogenital infections, and Lactobacillus probiotics to restore and/or maintain vaginal health has been advocated. These can interfere with the adherence, growth, and colonization by uropathogenic bacteria, thus reducing the risk of urinary tract infection (UTI). This study aims to isolate and evaluate the susceptibility of healthy vaginal and probiotic Lactobacillus spp. to urinary antibiotics.

Materials and methods A total of 50 premenopausal, nonmenstruating women with no symptoms of vaginal infection or UTI or antimicrobial use in the past 2 weeks were enrolled. Two high vaginal swabs were collected for Nugent's scoring and anaerobic culture. Colonies yielding gram-positive rods were confirmed by matrix-assisted laser desorption/ionization time-of-flight mass spectrometry (MALDI-TOF MS). Lactobacillus strains in a vaginal probiotic tablet were also isolated, and the sensitivity of both sets to urinary antibiotics was determined.

Results A total of 29 Lactobacillus spp. were isolated, including L. crispatus (15), L. gasseri (5), L. vaginalis (4), L. paracasei (2), L. curvatus (1), L. fermentum (1), and L. paraplantarum (1). All strains were susceptible to nitrofurantoin and resistant to norfloxacin, gentamicin, and cotrimoxazole (except $L$. paracasei). The probiotic strains

Keywords

- Lactobacillus

- probiotics

- UTI

- MALDI-TOF were pan-sensitive.

Conclusions Prophylactic antibiotics are capable of eliminating the normal vaginal inhabitants, which may increase the probability of UTI. The administration of vaginal probiotics as an alternate or multidrug therapy can restore vaginal microbiota and help prevent recurrent UTI.
\end{abstract}

\section{Introduction}

The vaginal microbiome has evolved to protect women against a variety of urogenital infections. Healthy vaginal microbiota is mainly dominated by Lactobacillus species, namely L. crispatus, L. gasseri, L. jensenii, and L. iners. ${ }^{1}$ Depletion of Lactobacilli may result in bacterial vaginosis (BV), which is associated with urinary tract infections (UTIs), increased risk of sexually transmitted infections, and pelvic inflammatory disease. ${ }^{2}$ UTI is a common problem among young healthy women of reproductive age group, with approximately 25 to 30\% reporting recurrence. Recurrent UTI (RUTI) is defined as at least three episodes of UTI in 12 months or at least two episodes in 6 months. Escherichia coli is the predominant pathogen in UTIs followed by Staphylococcus saprophyticus, Enterococcus faecalis, Klebsiella pneumoniae, and Proteus mirabilis. Antibiotics are effective in the treatment of UTIs but lead to increased antibiotic resistance in microorganisms. ${ }^{3}$ Antibiotic prophylaxis with agents such as cotrimoxazole, nitrofurantoin, and fluoroquinolones has been 
associated with a decreased rate of recurrence, from 2 to 3 cases per patient-year to 0.1 to 0.2 cases per patient-year, but it disrupts normal flora of urinary tract and increases development of resistance in uropathogens. ${ }^{4}$ The emergence of extended-spectrum $\beta$-lactamases and carbapenem-resistant E. coli emphasize the need to explore novel agents that have fewer side effects and promise long-term benefit. ${ }^{5}$ In case of RUTIs, disruption of normal vaginal flora has been shown to predispose patients to chronic intermittent urogenital infections. ${ }^{6}$ In this context, researchers have reported that the use of probiotics as an adjunct to antibiotic therapy may provide higher antimicrobial activity and reduce the dose of antibiotics required in addition to replenishing the intestinal flora and decreasing antibiotic side effects. ${ }^{5}$ Use of Lactobacilluscontaining probiotics to restore commensal vaginal flora has been proposed for the treatment and prophylaxis of bacterial urogenital infections. Florisia (CD Pharma India Pvt. Ltd.) is an intravaginal tablet containing at least $10^{9} \mathrm{CFU}$ (colonyforming unit) of lyophilized L. brevis, L. salivarius subsp. salicinus, and $L$. plantarum. The three strains have been selected for their ability to adhere to vaginal epithelial cells, production of $\mathrm{H}_{2} \mathrm{O}_{2}$, and coaggregation with pathogens. ${ }^{2}$ This study aims to isolate and identify the Lactobacillus species from the vagina of healthy females and probiotic tablet and to evaluate their susceptibility to urinary antibiotics.

\section{Materials and Methods}

This study was conducted from December 2016 to December 2017 with approval of the Institutional Ethical Committee and informed consent of all participants. A total of 50 premenopausal, nonmenstruating females (18-45 years old) who had no symptoms of UTI or vaginal infection were enrolled. Women with chronic autoimmune or inflammatory conditions or on oral/topical antimicrobials in the past 2 weeks were excluded. Two high vaginal swabs were collected from each female. Gram stain of direct smear was performed and graded on a 10-point scale based on the presence of Lactobacilli and other anaerobes as described by Nugent. The vaginal flora was defined as "healthy" if the score was 0 to 3, "intermediate" if 4 to 6 , and "BV" if $>7$. $^{7}$ Only women diagnosed as "healthy" were included in the analyses. The second swab from these "healthy" women was inoculated onto de Man, Rogosa, and Sharpe (MRS) and Brain Heart Infusion agar plates (HiMedia Laboratories, Mumbai, India). Plates were incubated anaerobically for 24 hours at $37^{\circ} \mathrm{C}$ in anaerobic jars with gaspak. ${ }^{8}$ White, mucoid, catalase-negative colonies on MRS agar yielding gram-positive rods were isolated for confirmation by matrix-assisted laser desorption/ionization time-of-flight mass spectrometry (MALDI-TOF MS, Bruker Daltonics, Hamburg, Germany). The probiotic tablet was incubated anaerobically at $37^{\circ} \mathrm{C}$ in MRS broth for 48 hours and then isolation on MRS agar was performed. Antimicrobial susceptibility of Lactobacillus isolates from healthy females and probiotic strains to antibiotics co-trimoxazole, norfloxacin, nitrofurantoin and gentamicin was determined by the method of Bauer et al using MRS agar under anaerobic conditions. ${ }^{9}$

\section{Results}

Of the participants, $66 \%(n=33)$ had Nugent's scores of 0 to 3 (-Table 1). A total of 29 species of Lactobacillus were isolated from these: most common being $L$. crispatus $(n=15)$ followed by $L$. gasseri $(n=5)$ and $L$. vaginalis $(n=4)$. The other species isolated were $L$. paracasei $(n=2)$, L. curvatus $(n=1)$, L. fermentum $(n=1)$, and $L$. paraplantarum $(n=1)$. All these isolates were susceptible to nitrofurantoin and resistant to norfloxacin and gentamicin. All the isolates were resistant to cotrimoxazole except $L$. paracasei. The probiotic tablet yielded L. brevis, L. salivarius, and L. plantarum, which were susceptible to all antibiotics.

\section{Discussion}

Lactobacilli are the predominant bacteria in vaginal flora and possess antimicrobial properties that regulate other urogenital microbiota. ${ }^{6}$ Most UTIs are caused by intestinal bacteria that ascend through the urethra to the bladder and, sometimes, kidneys. ${ }^{3}$ The Lactobacillus-dominated vaginal flora in premenopausal women impedes colonization of uropathogens due to competitive exclusion and maintaining low vaginal $\mathrm{pH} .{ }^{5}$ Inverse association has been reported between $\mathrm{H}_{2} \mathrm{O}_{2}$-producing Lactobacilli and vaginal E. coli colonization in women with RUTIs. Specific Lactobacilli strains can interfere with the adherence, growth, and colonization of uropathogenic bacteria and thus reduce the risk of ascent into the bladder. ${ }^{10}$ There are only a few studies characterizing the vaginal Lactobacillus species in healthy women of reproductive age in India (-Table 2). More studies in India are warranted as the species distribution in Indian women may be different. This would also guide as to whether replacement by a single species is sufficient or a balanced mixture depending on the local flora is needed in a probiotic.

The species most frequently isolated in our study was L. crispatus, which is a strong $\mathrm{H}_{2} \mathrm{O}_{2}$ producer and is associated with healthy vaginal microbiota. In addition, L. vaginalis was isolated, which has been associated with healthy flora. The other species was L. gasseri, and females carrying this are reported to have higher Nugent scores, though asymptomatic. ${ }^{14}$ Women in different geographical settings face different environmental conditions, dietary habits, and lifestyle. ${ }^{1}$ The results of our study resemble the most prevalent vaginal species in other Indian studies (-Table 2). Several authors have reported varying observations of vaginal Lactobacilli elsewhere in the world. ${ }^{14-17}$ Despite reports of high incidence of $L$. iners in human vaginal microbiota, we did not obtain isolates belonging to this species probably because of stringent nutritional requirements, very low oxygen tolerance, and molecular-based identification. ${ }^{15}$

Table 1 Nugent score of participants

\begin{tabular}{|l|l|l|}
\hline Nugent score & Category & Participants (\%) \\
\hline $0-3$ & Healthy & $33(66 \%)$ \\
\hline $4-6$ & Intermediate & $10(20 \%)$ \\
\hline$>7$ & Bacterial vaginosis & $7(14 \%)$ \\
\hline
\end{tabular}


Table 2 Comparison of Lactobacillus spp. isolated in various studies from India

\begin{tabular}{|c|c|c|c|c|c|}
\hline & Garg et al ${ }^{11}$ & $\begin{array}{l}\text { Madhivanan } \\
\text { et } \text { al }^{8}\end{array}$ & $\begin{array}{l}\text { Madhivanan } \\
\text { et al }\end{array}$ & $\begin{array}{l}\text { Pramanick } \\
\text { et } \text { al }^{13}\end{array}$ & $\begin{array}{l}\text { Das Purkayastha } \\
\text { et al }{ }^{1}\end{array}$ \\
\hline & $n=80$ & $n=11$ & $n=39$ & $n=107$ & $n=26$ \\
\hline Lactobacillus reuteri & $33 \%$ & $9 \%$ & $23 \%$ & $22 \%$ & $1 \%$ \\
\hline Lactobacillus fermentum & $25 \%$ & $9 \%$ & $15 \%$ & - & $6 \%$ \\
\hline Lactobacillus salivarius & $16 \%$ & $9 \%$ & - & - & - \\
\hline Lactobacillus crispatus & $5 \%$ & $27 \%$ & $41 \%$ & $27 \%$ & - \\
\hline Lactobacillus gasseri & $3 \%$ & - & $46 \%$ & $15 \%$ & $4 \%$ \\
\hline Lactobacillus jensenii & $4 \%$ & $27 \%$ & $18 \%$ & $16 \%$ & $2 \%$ \\
\hline Lactobacillus iners & - & - & - & $65 \%$ & - \\
\hline Lactobacillus mucosae & - & - & $5 \%$ & - & $16 \%$ \\
\hline
\end{tabular}

Several in vitro and in vivo studies support the beneficial effect of Lactobacilli on the restoration of vaginal flora and the prevention of RUTIs. The rationale for the use of probiotics is based on the regulatory role played by commensal microflora in the gastrointestinal and genitourinary tracts. ${ }^{6}$ In their meta-analysis, Grin et al concluded that probiotic strains of Lactobacillus are safe and effective in preventing RUTI in adult women. ${ }^{18}$ Barrons and Tassone concluded that intravaginal Lactobacillus suppositories are effective against uropathogens and show the greatest efficacy for UTI prophylaxis. ${ }^{6}$ In another study, the use of Lactobacillus vaginal suppository in women resulted in the reduction of $E$. coli positive cultures from $5.0 \pm 1.6$ episodes to $1.3 \pm 1.2$ episodes $(p<0.0007)$ over a 12 -month period. ${ }^{19}$

Prolonged low-dose chemotherapeutic agents such as nitrofurantoin, ciprofloxacin, trimethoprim, and cotrimoxazole have been traditionally used as prophylaxis in reducing UTI. ${ }^{3}$ All Lactobacillus isolates were found resistant to cotrimoxazole except the $L$. paracasei strains. All strains were susceptible to nitrofurantoin and resistant to norfloxacin and gentamicin. The three probiotic strains were found susceptible to all the tested antibiotics. This suggests that prophylactic antibiotics are capable of eliminating the normal vaginal inhabitants, which are the main inhibitors of urinary pathogens. This way they counteract the benefits of antibacterial effect and increase the probability of UTI.

\section{Conclusion}

We suggest that the administration of vaginal probiotics can restore vaginal microbiota and help prevent recurrence of UTI, particularly as part of an alternate therapy or multidrug treatment. The limitation of our study is the small sample size. Also, as our methodology was culture-based, only those Lactobacillus species that can be cultured were identified. To the best of our knowledge, very few similar studies have been performed in India, and large-scale studies in future would help in generating conclusive evidence for alternative therapies.

\section{Ethical Approval}

All procedures performed in this study were in accordance with the ethical standards of the institutional and/or national research committee and with the 1964 Helsinki declaration and its later amendments or comparable ethical standards.

\section{Funding}

This study was part of a research project funded by the Department of Science and Technology, Government of India.

\section{Presentation at a Meeting}

North-West Chapter of the Indian Association of Medical Microbiologists, Rohtak, Haryana, India.

\section{Conflict of Interest}

Dr. Chander reports grants from the Department of Science and Technology, Government of India, during the conduct of the study. Dr. V. Gupta reports grants from the Department of Science and Technology, Government of India, during the conduct of the study. Dr. Goel reports grants from the Department of Science and Technology, Government of India, during the conduct of the study. Dr. Singhal reports grants from the Department of Science and Technology, Government of India, during the conduct of the study. Dr. M. Gupta reports grants from the Department of Science and Technology, Government of India, during the conduct of the study.

\section{Acknowledgment}

The authors gratefully acknowledge the Department of Medical Microbiology, Postgraduate Institute of Medical Education and Research, Chandigarh, India, for their help in species identification by MALDI-TOF MS.

\section{References}

1 Das Purkayastha S, Bhattacharya MK, Prasad HK, et al. Contrasting diversity of vaginal Lactobacilli among the females of Northeast India. BMC Microbiol 2019;19(1):198

2 Mastromarino P, Hemalatha R, Barbonetti A, et al. Biological control of vaginosis to improve reproductive health. Indian $\mathrm{J}$ Med Res 2014;140(Suppl):S91-S97

3 Al-Badr A, Al-Shaikh G. Recurrent urinary tract infections management in women - a review. Sultan Qaboos Univ Med J 2013;13(3):359-367 
4 Reid G, Bruce AW, Cook RL, Llano M. Effect on urogenital flora of antibiotic therapy for urinary tract infection. Scand J Infect Dis 1990;22(1):43-47

5 Gupta V, Nag D, Garg P. Recurrent urinary tract infections in women: how promising is the use of probiotics? Indian J Med Microbiol 2017;35(3):347-354

6 Barrons R, Tassone D. Use of Lactobacillus probiotics for bacterial genitourinary infections in women: a review. Clin Ther 2008;30(3):453-468

7 Nugent RP, Krohn MA, Hillier SL. Reliability of diagnosing bacterial vaginosis is improved by a standardized method of gram stain interpretation. J Clin Microbiol 1991;29(2):297-301

8 Madhivanan P, Raphael E, Rumphs A, et al. Characterization of culturable vaginal Lactobacillus species among women with and without bacterial vaginosis from the United States and India: a cross-sectional study. J Med Microbiol 2014;63(Pt 7) : 931-935

9 Bauer AW, Kirby WM, Sherris JC, Turck M. Antibiotic susceptibility testing by a standardized single disk method. Am J Clin Pathol 1966;45(4):493-496

10 Gupta K, Stapleton AE, Hooton TM, Roberts PL, Fennell CL, Stamm WE. Inverse association of H2O2-producing Lactobacilli and vaginal Escherichia coli colonization in women with recurrent urinary tract infections. J Infect Dis 1998;178(2): $446-450$

11 Garg KB, Ganguli I, Das R, Talwar GP. Spectrum of Lactobacillus species present in healthy vagina of Indian women. Indian J Med Res 2009;129(6):652-657
12 Madhivanan P, Alleyn HN, Raphael E, et al. Identification of culturable vaginal Lactobacillus species among reproductive age women in Mysore, India. J Med Microbiol 2015;64(6):636-641

13 Pramanick R, Parab S, Mayadeo N, Warke H, Aranha C. Cross sectional analysis of vaginal Lactobacillus in asymptomatic women of reproductive age in Mumbai, India. J Infect Dev Ctries 2018;12(12):1096-1104

14 Vásquez A, Jakobsson T, Ahrné S, Forsum U, Molin G. Vaginal Lactobacillus flora of healthy Swedish women. J Clin Microbiol 2002;40(8):2746-2749

15 Pino A, Bartolo E, Caggia C, Cianci A, Randazzo CL. Detection of vaginal Lactobacilli as probiotic candidates. Sci Rep 2019;9(1):3355

16 Zhang R, Daroczy K, Xiao B, Yu L, Chen R, Liao Q. Qualitative and semiquantitative analysis of Lactobacillus species in the vaginas of healthy fertile and postmenopausal Chinese women. J Med Microbiol 2012;61(Pt 5) :729-739

17 Pendharkar S, Magopane T, Larsson PG, et al. Identification and characterisation of vaginal Lactobacilli from South African women. BMC Infect Dis 2013;13:43

18 Grin PM, Kowalewska PM, Alhazzan W, Fox-Robichaud AE. Lactobacillus for preventing recurrent urinary tract infections in women: meta-analysis. Can J Urol 2013;20(1):6607-6614

19 Uehara S, Monden K, Nomoto K, Seno Y, Kariyama R, Kumon $\mathrm{H}$. A pilot study evaluating the safety and effectiveness of Lactobacillus vaginal suppositories in patients with recurrent urinary tract infection. Int $\mathrm{J}$ Antimicrob Agents 2006;28(Suppl 1) :S30-S34 